\title{
A deep survey for Galactic Wolf-Rayet stars. II. Implications for Galactic structure and massive star formation
}

\author{
Michael M. Shara ${ }^{1}$, Michael Potter ${ }^{1}$, Anthony F.J. Moffat ${ }^{2}$, Robert \\ Lamontagne, Lindsey F. Smith, and Virpi S. Niemela ${ }^{4}$ \\ ${ }^{1}$ Space Telescope Science Institute, Baltimore, USA \\ ${ }^{2}$ Départment de Physique, Université de Montréal, Canada \\ ${ }^{3}$ School of Physics, University of Sydney, Australia \\ ${ }^{4}$ Facultad de Ciencias Astronómicas y Geofísicas, \\ Universidad Nacional de La Plata, Argentina
}

\section{Introduction}

We have completed a direct narrow-band/broad-band Schmidt-plate survey of large areas of the southern Milky Way for new Wolf-Rayet stars as faint as 19th $b$ magnitude. The 31 newly detected stars in the completed survey are amongst the reddest and/or most distant known in the Galaxy. We have obtained spectra of all candidate WR stars in the 22 fields stretching from $l=282^{\circ}$ to $l=341^{\circ}$ in longitude, and $\Delta b=3.5^{\circ}$ in latitude, covering about 180 square degrees. We also observed two isolated Milky Way fields centered at $l=0^{\circ}$ and $l=8^{\circ}$. Eighteen new WR stars are reported here for the first time. Combined with the 13 new WR stars we have already reported in Carina, our list of 31 new Galactic WR stars reaches 3-4 magnitudes fainter than previous surveys. Thirteen of the 18 new WR stars reported here are of subtype WN, while five are of subtype WC. Our new WR stars clearly demonstrate an increasing WN/WC number ratio with increasing Galactocentric distance.

\section{Observations and poster display}

The prime motivation and scientific driver of this survey is to search for the predicted WC/WN ratio gradient in the Galaxy. To do this we must push the completeness of Galactic WR stars to much fainter magnitudes than has yet been achieved. Our preliminary results (Shara et al. 1991) were the detection of 13 new WR stars in 40 square degrees in Carina, where 19 WR stars were previously known. Eleven new WN stars and only two WC stars were found, supporting the Maeder (1991) scenario of $Z$-controlled WN/WC ratio. A significantly larger areal coverage and sample of WR stars are needed to strengthen or refute our early conclusions, and to more rigorously test the Maeder(1991) scenario. This has largely been achieved: the Galactic WC/WN ratio is clearly observed to be monotonically increasing with decreasing Galactocentric distance (and hence with increasing metallicity). 
Galactic WC/WN Gradient at Various R in Contiguous Survey Region

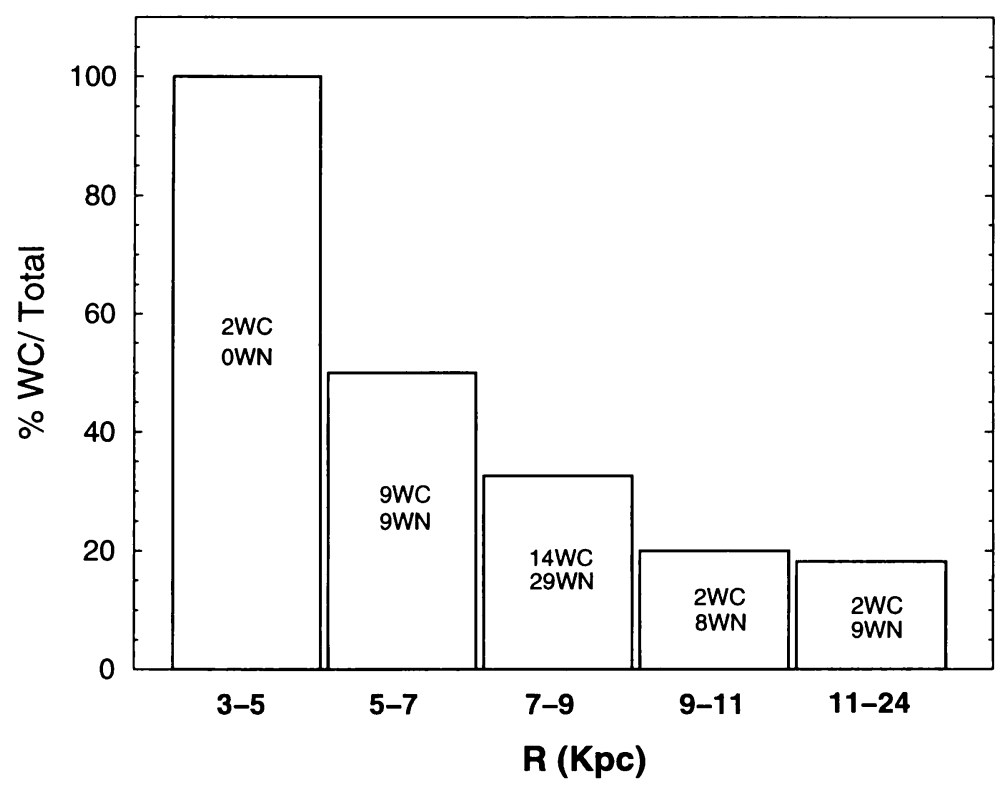

\section{References}

Maeder, A. 1991, A\&A 242, 93

Shara, M.M., Smith, L.F., Potter, M., Moffat, A.F.J. 1991, AJ 102, 716

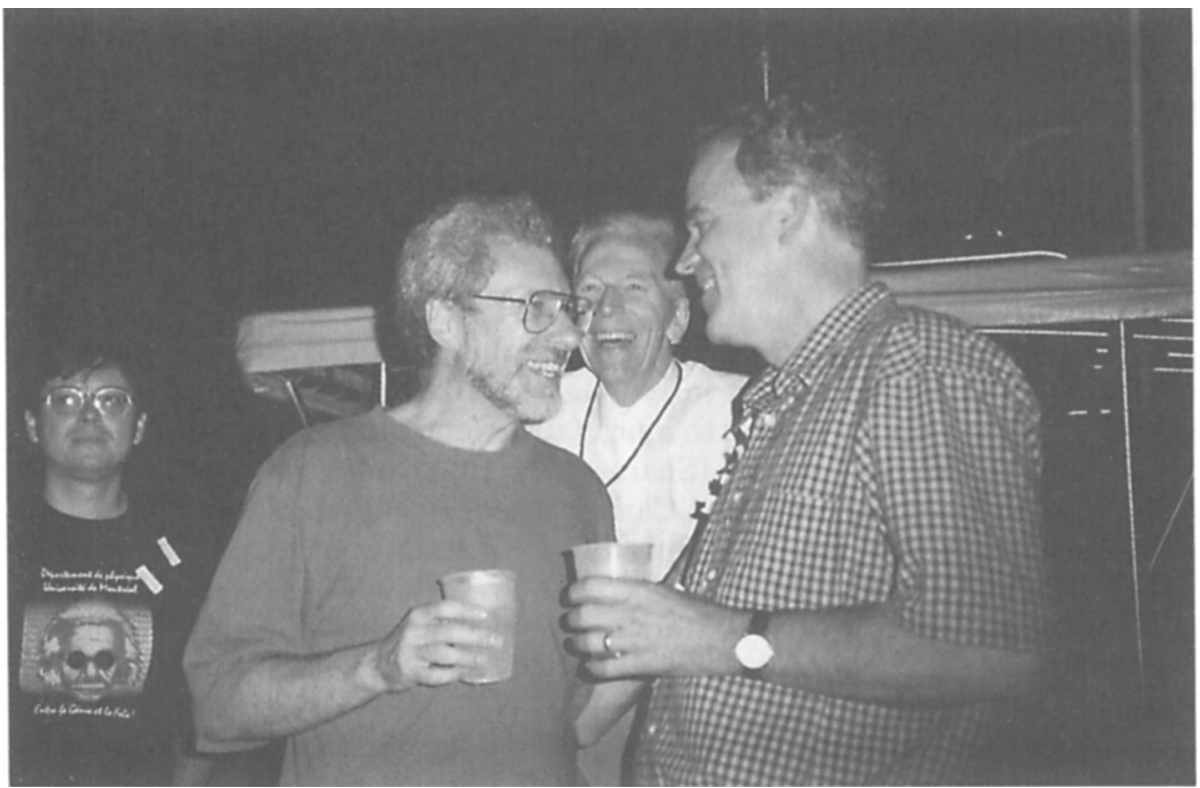

